\title{
Insulin sensitivity, proinsulin and insulin as predictors of coronary heart disease. A population-based 10-year, follow-up study in 70-year-old men using the euglycaemic insulin clamp
}

Received: 9 August 2004 / Accepted: 12 December 2004 / Published online: 1 April 2005

C) Springer-Verlag 2005

\begin{abstract}
Aims/hypothesis: The association between CHD and insulin sensitivity ( $\mathrm{Si}$ ) measured by the euglycaemic insulin clamp has not been examined previously. Earlier studies found a relationship between CHD and elevated plasma insulin, an analysis that may have been confounded by co-determination of proinsulin, which has evolved as a stronger predictor of CHD. The aim was to determine the longitudinal relationships between $\mathrm{S} i$, intact proinsulin, 32-33 split proinsulin, specific insulin and subsequent CHD. Methods: This was a population-based cohort study of 815 men in Uppsala, Sweden, aged 70 years at baseline with a follow-up of up to 10 years. Baseline insulin sensitivity was determined by euglycaemic insulin clamp. Fasting proinsulin, 32-33 split proinsulin and specific insulin concentrations were analysed using specific two-site immunometric assays. CHD was taken as diagnosed, if stated (in the event of death) on the Cause of Death Registry, or for subjects hospitalised for the first time with CHD, if CHD was recorded in the Hospital-Discharge Registry. The associations were analysed using Cox's proportional hazards, presented as hazard ratios (HRs) with their $95 \%$ CIs for a one-SD increase in the predictor. Results: In multivariate analysis, Si (HR:0.80, CI:0.65-0.97) adjusted for serum cholesterol, systolic blood pressure, fasting plasma glucose, BMI and smoking predicted CHD. Intact proinsulin (HR:1.18, CI:1.01-1.38), adjusted as the model above, predicted CHD, whereas 32-33 split proinsulin (HR:1.13,
\end{abstract}

\author{
B. Zethelius $(\triangle) \cdot$ H. Lithell \\ Department of Public Health and Caring Sciences/Geriatrics, \\ P.O. Box 609, 75125 Uppsala, Sweden \\ e-mail: bjorn.zethelius@pubcare.uu.se \\ Tel.: +46-18-6117981 \\ Fax: +46-18-6117976 \\ C. N. Hales \\ Department of Clinical Biochemistry, \\ Addenbrooke's Hospital, \\ Cambridge, UK \\ C. Berne \\ Department of Medical Sciences, \\ Uppsala University, \\ Uppsala, Sweden
}

CI:0.95-1.35) or specific insulin (HR:1.07, CI:0.89-1.30) did not. Conclusions/interpretation: Insulin resistance measured by the euglycaemic insulin clamp predicts subsequent CHD in elderly men. Proinsulin provides a better prediction of CHD than insulin.

Keywords Coronary heart disease · Insulin · Predictor . Proinsulin · Risk factors

Abbreviations CVD: cardiovascular disease -

HOMA-IR: Homeostasis model assessment of insulin resistance - HR: Hazard ratio - IRI: immunoreactive insulin PAI-1: Plasminogen activator inhibitor-1 . PYAR: person years at risk $\cdot \mathrm{S} i$ : Insulin sensitivity index

\section{Introduction}

Type 2 diabetes mellitus, hypertension and dyslipidaemia are associated with insulin resistance and an increased risk of CHD [1]. Atherosclerosis is associated with insulin resistance [2], but the extent to which insulin resistance contributes to the risk of subsequent CHD has so far mostly been studied by using the plasma immunoreactive insulin (IRI) concentration as a surrogate marker of insulin resistance or by the minimal model technique [2].

The plasma IRI concentration correlates only moderately to insulin sensitivity when measured with the gold standard euglycaemic insulin clamp technique [3]. In addition, the role of IRI as a risk factor for CHD has been cast in doubt $[4,5]$. Even if IRI is accepted as a marker for cardiovascular disease, it is weak compared with established risk markers $[6,7]$. Also, the length of follow-up attenuates the strength of IRI as a risk marker [8]. Further, the strength of various risk factors for CHD may change between middle-aged and elderly patients [9].

Proinsulin, in contrast to specific insulin, was recently shown to be a long-term predictor of CHD in middle-aged men in the UK [10] and in Sweden [11]. Also, in studies with a shorter follow-up, proinsulin predicts myocardial infarction [12] and first-ever stroke [13]. 
The aim of this study was to investigate, using data from the ULSAM cohort (Uppsala Longitudinal Study of Adult Men) with baseline at age 70 , whether insulin-mediated glucose uptake measured with the euglycaemic insulin clamp [14], intact and 32-33 split proinsulin and true insulin measured by specific methods [15] are predictive of CHD in elderly males with up to 10 years of follow-up, and whether such associations are influenced by conventional risk factors.

\section{Subjects, materials and methods}

Study population In 1970, all men born from 1920 to 1924 and residing in Uppsala were invited to a health survey, in which $82 \%(n=2,322)$ participated [16]. After 20 years, at the age of 70, they were invited for re-investigation, which formed the baseline of the present study, comprising 1,221 of the 1,681 men who were still alive (73\%) [17]. Complete data from the baseline investigation at age 70 years were available from 1,166 subjects.

In order to select subjects free from cardiovascular disease (CVD) at baseline, 351 men were excluded due to the presence of: (1) prior myocardial infarction or angina pectoris; (2) Q or QS-complexes or left bundle branch block (Minnesota codes $1.1-1.3$ or 7.1, respectively) in the baseline ECG-registration; and (3) previous CVD (International Classification of Diseases, 9th revision [ICD-9] codes: $390-459$, equivalent to 10 th revision [ICD-10] codes I00I99) or incident CVD up to 1 year after baseline $(n=4)$ to avoid subclinical CVD or current treatment with nitrates or cardiac glycosides. Subjects identified with diabetes at baseline were excluded, as fasting concentrations of insulin are to a varying extent affected by the diabetic state, with elevated concentrations driven by insulin resistance in early type 2 diabetes or by insulinopenia, due to later beta cell failure. After these exclusions, the main analysis in total comprised 815 subjects.

Subjects' medical histories and ongoing current pharmacological treatments were collected using the original protocol questionnaire [16]. CHD mortality and morbidity data were collected from the official Swedish registries held by The Centre for Epidemiology, National Board of Health and Welfare in Sweden.

Informed consent and ethics committee approval The Ethics Committee of the Faculty of Medicine at Uppsala University approved the study. Written informed consent was obtained from all subjects.

Follow-up data CHD was defined on the basis of cause of death recorded in the Cause of Death Registry, or for patients first hospitalised for CHD on the basis of diagnoses (ICD-9 codes:410-414, ICD-10 codes I20-25) recorded in the Hospital-Discharge Registry (censor date December 31, 2001). CHD morbidity, defined by combining data from the Cause of Death Registry and the Hospital Discharge Registry, is an efficient, validated alternative to revised hospital discharge notes and death certificates [18, 19]. No subject was lost to follow-up due to missing registry data.

Euglyacemic insulin clamp For measurement of insulin sensitivity [14], insulin was infused at a constant rate of 56 $\mathrm{mU} \cdot \mathrm{min}^{-1} \cdot \mathrm{m}^{-2}$ calculated to achieve nearly complete suppression of hepatic glucose output. The target level of plasma glucose (measured every 5th minute during the 2-h clamp) was $5.1 \mathrm{mmol} / \mathrm{l}$. Median was $5.1 \mathrm{mmol} / \mathrm{l}$, 5 th percentile $5.0 \mathrm{mmol} / 1,95 \%$ percentile $5.4 \mathrm{mmol} / \mathrm{l}$ and mean $( \pm \mathrm{SD}) 5.2( \pm 1.3) \mathrm{mmol} / \mathrm{l}$. The insulin sensitivity index $(\mathrm{S} i)$ was calculated as glucose disposal rate (glucose infused in $\mathrm{mg} \cdot \mathrm{min}^{-1} \cdot \mathrm{kg}$ body weight ${ }^{-1}$ ) divided by the mean plasma insulin concentration per $100 \mathrm{mU} / \mathrm{l}$ during the last $60 \mathrm{~min}$ of the 2-h euglycaemic insulin clamp.

Baseline characteristics Blood samples were drawn in the morning after an overnight fast. The plasma concentrations of intact proinsulin and 32-33 split proinsulin were analysed using the two-site immunometric assay technique [15] and concentrations of specific insulin by the Access Immunoassay System (Sanofi Pasteur Diagnostics), blinded for outcome, at the Department of Clinical Biochemistry, Addenbrooke's Hospital, Cambridge, UK. Assays are highly specific and cross reactivity was less than $1 \%$ with insulin in the intact proinsulin assay [15]. IRI was determined by the Phadebas insulin test (Pharmacia, Uppsala, Sweden) [20].

Type 2 diabetes mellitus and IGT were defined according to the WHO 1999 criteria [21] using a 75-g OGTT or the use of oral hypoglycaemic agents. Fasting concentrations of plasma glucose and serum cholesterol, weight, height, BMI and an ECG, supine systolic and diastolic blood pressures were measured under standardised conditions [17, 20]. Smoking status was taken from the questionnaire.

Statistical analyses Skewed variables were log-transformed to achieve normal distribution. Normally distributed variables were used in all statistical analyses. The statistical software package STATA 6.0 for PC (STATA, College Station, TX, USA) was used. All tests were two-tailed and a $p$-value of less than 0.05 was considered significant. Associations between baseline variables were analysed using Pearson product moment correlations. In the prospective analyses, Cox's proportional hazard regression analyses were used. Hazard ratios (HRs) with two-tailed 95\% CIs were investigated for a one standard deviation increase in a continuous variable and for a one step increase in the dichotomous variable smoking, to determine the magnitude of the relationship to, and the statistical significance of the predictors of the defined outcome. Kaplan-Meier plots were performed to confirm proportionality of hazards. No violations of the proportional assumption were found using Schoenfeld's residuals in a linear correlation test for each Cox model presented. In the multivariate CHD models, adjustments were made for the following risk factors: serum cholesterol, systolic blood pressure, fasting plasma glucose, BMI and smoking. 


\section{Results}

Baseline clinical characteristics at age 70 and standardised crude HRs for a first CHD event during follow-up are shown in Table 1. The subjects had a median follow-up time of 7.9 years (maximum 10.4 years), with a total of 5,972 person-years at risk (PYAR). During follow-up, 126 of 815 subjects (rate 2.11/100 PYAR) had a CHD event, of whom 51 died. The "overlap" of CHD cases between this study and our previous study of the ULSAM-cohort [11] with baseline at age 50 instead of age 70 as in the present study was only 12 out of 126 cases $(9.5 \%)$. IGT $(n=214 / 815)$ did not predict CHD (HR:1.18; 95\% CI:0.82-1.69; $p=0.36$ ).

$\mathrm{S} i$, intact and 32-33 split proinsulin, serum cholesterol, smoking, systolic blood pressure, fasting glucose and BMI were all associated with a subsequent CHD event in the univariate analyses (Table 1), whereas specific insulin, IRI or diastolic blood pressure were not.

Unadjusted Kaplan-Meier survival curves showed that the risk of CHD was higher for men in the lowest tertile compared to men in the highest tertile of $\mathrm{S} i(<3.6 \mathrm{vs}>5.8$, $p<0.001$; Fig. 1a). For plasma proinsulin concentrations,

Table 1 Clinical characteristics at baseline and crude hazard ratios for incident coronary heart disease $(n=126)$, over the 10.4 years of follow-up

\begin{tabular}{|c|c|c|}
\hline Characteristic & $\begin{array}{l}\text { Mean } \pm \text { SD } \\
(n=815)\end{array}$ & $\begin{array}{l}\text { Hazard ratio } \\
(95 \% \mathrm{CI}) p\end{array}$ \\
\hline $\begin{array}{l}\mathrm{S} i\left(\mathrm{mg} \cdot \mathrm{min}^{-1} \cdot \mathrm{kg}^{-1} /\right. \\
[100 \mathrm{mU} / \mathrm{l}])\end{array}$ & $5.1 \pm .2 .5$ & $\begin{array}{l}0.76(0.63-0.92) \\
0.003\end{array}$ \\
\hline Intact proinsulin (pmol/1) & $8.4 \pm 8.4$ & $\begin{array}{l}1.24(1.07-1.45) \\
0.005\end{array}$ \\
\hline $\begin{array}{l}\text { 32-33 split proinsulin } \\
(\mathrm{pmol} / \mathrm{l})\end{array}$ & $11.0 \pm 12.4$ & $\begin{array}{l}1.19(0.99-1.41) \\
0.058\end{array}$ \\
\hline Specific insulin $(\mathrm{pmol} / \mathrm{l})$ & $53.2 \pm 47.9$ & $\begin{array}{l}1.07(0.89-1.28) \\
0.476\end{array}$ \\
\hline IRI (pmol/l) & $76.8 \pm 51.3$ & $\begin{array}{l}1.13(0.94-1.36) \\
0.162\end{array}$ \\
\hline $\begin{array}{l}\text { Serum cholesterol } \\
(\mathrm{mmol} / \mathrm{l})\end{array}$ & $5.8 \pm 1.0$ & $\begin{array}{l}1.18(1.00-1.48) \\
0.048\end{array}$ \\
\hline Smoking $(\%)$ & 19.7 & $\begin{array}{l}1.75(1.19-2.58) \\
0.004\end{array}$ \\
\hline $\begin{array}{l}\text { Systolic blood pressure } \\
(\mathrm{mmHg})\end{array}$ & $146 \pm 18$ & $\begin{array}{l}1.33(1.13-1.58) \\
0.001\end{array}$ \\
\hline $\begin{array}{l}\text { Diastolic blood pressure } \\
(\mathrm{mmHg})\end{array}$ & $84 \pm 9$ & $\begin{array}{l}1.11(0.93-1.34) \\
0.218\end{array}$ \\
\hline $\begin{array}{l}\text { Fasting plasma glucose } \\
(\mathrm{mmol} / \mathrm{l})\end{array}$ & $5.0 \pm 0.6$ & $\begin{array}{l}1.20(1.03-1.41) \\
0.015\end{array}$ \\
\hline Body mass index $\left(\mathrm{kg} / \mathrm{m}^{2}\right)$ & $26.2 \pm 3.3$ & $\begin{array}{l}1.22(1.02-1.47) \\
0.025\end{array}$ \\
\hline
\end{tabular}

Unless otherwise stated, data are shown as arithmetic means $\pm \mathrm{SD}$, hazard ratios with $95 \%$ CIs and $p$-value. Cox's proportional hazards regression was applied to variables standardised to $1 \mathrm{SD}$ (except smoking). Si, insulin sensitivity index derived from euglycaemic insulin clamp; IRI, immunoreactive insulin; CHD, coronary heart disease (ICD-9 codes 410-414, ICD-10 codes I20-25)
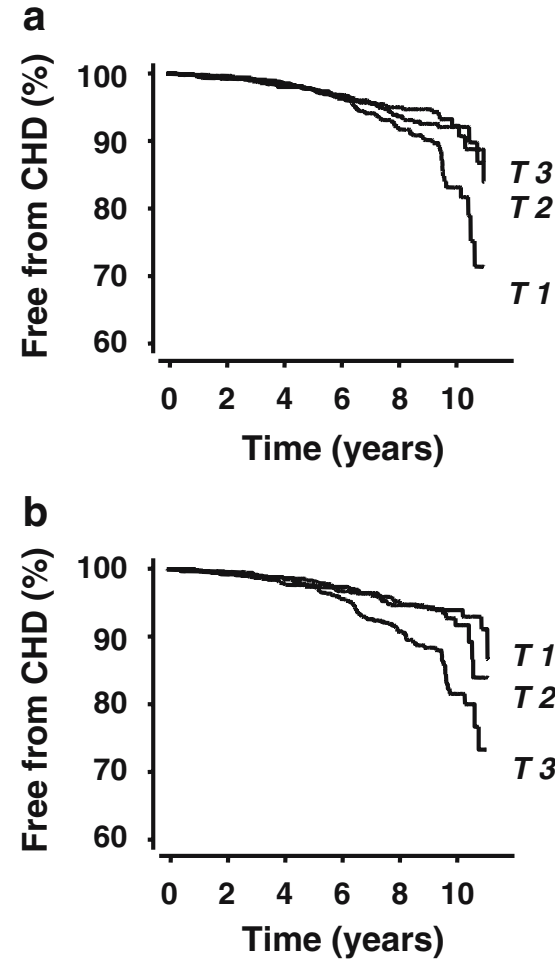

Fig. 1 Kaplan-Meier survival curves for subjects free from subsequent CHD events during 10.4 years of follow-up, for a tertiles of insulin sensitivity index $(\mathrm{S} i)$, and $\mathbf{b}$ tertiles of intact proinsulin at baseline. The risk of CHD was significantly higher for men in the lowest tertile (T1) of $\mathrm{S} i$ than for those in the highest tertile (T3), ( $\mathrm{S} i$ $<3.6$ vs $>5.8, p<0.001)$. Similarly, the risk of CHD was significantly higher for men in the highest tertile (T3) of proinsulin concentration than for those in the lowest tertile (T1), (proinsulin $>8.1 \mathrm{vs}<4.8 \mathrm{pmol} / \mathrm{l}$, $p<0.001$ )

the risk of CHD was higher in the highest tertile compared to the lowest tertile $(>8.1 \mathrm{pmol} / 1$ vs $<4.8 \mathrm{pmol} / 1, p<0.001$; Fig. 1b).

The association between $\mathrm{S} i$ and $\mathrm{CHD}$, adjusted for serum cholesterol, systolic blood pressure, fasting glucose, BMI and smoking in multivariate analysis, showed that a 1-SD increase in $\mathrm{S} i$ decreased the risk for a CHD event by $18 \%$ (Table 2, model 1).

In similar multivariate models, intact proinsulin predicted CHD independently of serum cholesterol, systolic blood pressure, fasting glucose, BMI and smoking (Table 2, model 2), in contrast to 32-33 split proinsulin or specific insulin or IRI (Table 2, models 3, 4 and 5). Combined models including $\mathrm{S} i$ and proinsulin or $\mathrm{S} i$ and insulin, where the associations for both with CHD became non-significant, were not valid due to co-linearity between $\mathrm{S} i$ and proinsulin or $\mathrm{S} i$ and insulin. The cross-sectional association with $\mathrm{S} i$ was stronger for plasma values of proinsulin $(r=-0.51$, $p<0.001)$ and insulin $(r=-0.62, p<0.001)$ than the association between early insulin response at an OGTT and proinsulin $(r=0.21, p<0.001)$ or insulin $(r=0.36, p<0.001)$. 
Table 2 Multivariate hazard ratios for incident coronary heart disease over the 10.4-years follow-up, according to insulin sensitivity, intact and 32-33 split proinsulin, specific insulin and IRI with adjustments for serum cholesterol, systolic blood pressure, fasting plasma glucose, body mass index and smoking

\begin{tabular}{ll}
\hline & Hazard ratio $(95 \% \mathrm{CI}) p$ \\
\hline $\begin{array}{l}\text { Model } 1 \\
\mathrm{~S} i\end{array}$ & $0.80(0.65-0.97) 0.026$ \\
Model 2 & $1.18(1.01-1.38) 0.039$ \\
Intact proinsulin & \\
Model 3 & $1.13(0.95-1.35) 0.172$ \\
32-33 split proinsulin & \\
Model 4 & $1.07(0.89-1.30) 0.467$ \\
Specific insulin & \\
Model 5 & $1.17(0.97-1.40) 0.094$ \\
Immunoreactive insulin &
\end{tabular}

Data are hazard ratios with $95 \%$ CIs and $p$ values. Cox's proportional hazards regression was applied to variables standardised to $1 \mathrm{SD}$ (except smoking). Si, insulin sensitivity index derived from euglycaemic insulin clamp; IRI, immunoreactive insulin; CHD, coronary heart disease (ICD-9 codes 410-414, ICD-10 codes I20-25)

\section{Discussion}

A low insulin-mediated glucose uptake measured by the euglycaemic insulin clamp technique [14] predicted subsequent CHD over a follow-up period up to 10 years in this population-based cohort study of 70-year old men and thus suggested that insulin resistance makes a major contribution to mortality and morbidity also in the elderly. The risk of subsequent CHD was independent of serum cholesterol, systolic blood pressure, fasting glucose, BMI and smoking, in contrast to a similar analysis of specific insulin or IRI, which are commonly used as surrogate markers for insulin sensitivity. Like insulin-mediated glucose uptake, serum proinsulin was independent of the conventional risk factors named above in predicting CHD.

The observation that decreased insulin-mediated glucose disposal, in contrast to specific insulin concentrations, predicted subsequent CHD indicates that the link between atherosclerosis and insulin resistance is more closely associated with impaired insulin action than with the compensatory increase of plasma insulin, which has been suggested to be atherogenic [22]. The weak association between plasma insulin and CHD is supported by evidence from 25 prospective epidemiological studies [6] and, for subjects with type 2 diabetes, evidence from the UKPDS [23]. In a meta-analysis [7] of 12 large-scale longitudinal studies, it was found that the association between IRI and CHD was modified by the type of insulin assay used. Recent studies using the homeostasis model assessment of insulin resistance (HOMA-IR) index as a surrogate marker of insulin sensitivity have also reported conflicting results [24, 25]. The variation in HOMA-IR is explained to a great extent by the variation in insulin [24]. In contrast to most previous epidemiological studies, which have measured IRI with assays that cross-react with proinsulin, our study avoided this problem by using assays specific for insulin and proinsulin.

Defective insulin action assessed by the euglycaemic insulin clamp is associated, more strongly than plasma insulin, with a well-established cluster of abnormalities, i.e. type 2 diabetes mellitus or IGT, hypertension, dyslipidaemia, and central obesity, which together comprise the insulin resistance syndrome [5]. Another abnormality that may play a role in promoting an increased risk of CHD is the minor elevation of plasma glucose that follows impaired insulin action and underlies hypersecretion of insulin and its propeptides [26]. Also strongly associated with insulin resistance are elevated plasma NEFA, which aggravate the insulin-resistant state both in skeletal muscle and in the liver, and also have direct vascular effects like promoting endothelial dysfunction $[27,28]$ and elevating blood pressure [29].

Consistent with previous studies in middle-aged men $[10,11,30]$, plasma proinsulin was a stronger predictor of CHD than insulin. High plasma proinsulin concentrations are associated with coronary artery atherosclerosis [31] and with increased intimal media thickness in the common carotid artery [32]. In clinical trials with human proinsulin, a several-fold increase was found in cardiovascular events in comparison with human insulin [33], which suggested that thrombo-embolic mechanisms had been activated [33]. In vitro, proinsulin stimulates production of plasminogen activator inhibitor-1 (PAI-1) [34, 35], a putative link between athero-thrombosis and elevated proinsulin concentrations preceding the onset of CHD [36]. The association between proinsulin and atherosclerosis in the common carotid artery also became attenuated by adjustment for PAI-1 in one study [32]. These findings suggest that proinsulin plays some role in biological mechanisms involving atherothrombosis. Another possibility suggested is that proinsulin-like molecules have actions via a proinsulin receptor [37]. With these data in mind, we have not used the term risk factor to describe the observed associations, but instead have chosen the term predictor (equivalent to risk marker) for proinsulin, as published observational studies [10-13] and the present study do not provide sufficient evidence to support a causal linkage.

Elevated plasma proinsulin, an indicator of beta cell hypersecretion, showed a strong association with the degree of insulin resistance. Both were found to be predictors of CHD in this cohort, but when included in the same model, their predictive capacity was weakened. Proinsulin, as a marker of insulin resistance and Si may therefore partly reflect different aspects of insulin resistance. Proinsulin and insulin concentrations are measured in the fasting state and could thus reflect hepatic insulin resistance to a greater extent than insulin-mediated glucose uptake during a euglycaemic insulin clamp, which mainly measures skeletal muscle glucose uptake at an insulin level at which hepatic glucose production is suppressed in the majority of subjects.

Type 2 diabetes mellitus was associated with CHD, in contrast to IGT, which is consistent with previous findings in elderly subjects in Finland [9]. Subjects with diabetes 
have an increased risk of CHD [38] and have elevated plasma proinsulin [39]. By excluding prevalent diabetes at baseline in our main analysis, we avoided confounding by co-morbidity of the relationship between proinsulin and CHD. The present demonstration that elevated serum proinsulin is a predictor of CHD also in the elderly non-diabetic population extends our previous report in middle-aged men [11] and one report on proinsulin as a predictor of CHD in middle-aged subjects in Britain [10].

At age 70, subjects with the insulin resistance syndrome [5] from the original cohort with baseline at age 50 and examined between 1970 and 1973 are likely to have escaped follow-up due to increased cardiovascular mortality before they were invited to the re-investigations that formed the baseline of the present study at age 70 . Thus, the magnitude of the observed associations between insulin resistance, proinsulin and CHD are lower at age 70 than in middle-aged subjects [11].

The present study is unable to answer the question of whether the prediction of CHD risk can also be applied to women. However, in another study of elderly subjects without type 2 diabetes and which included men and women, proinsulin was more strongly and consistently associated with CHD than specific insulin [40].

We conclude that insulin resistance determined by the euglycaemic insulin clamp in elderly men is a predictor of CHD, independently of conventional cardiovascular risk factors. Further, proinsulin provides better prediction of CHD than insulin as a surrogate risk marker for insulin resistance.

Acknowledgements This work was supported by research grants from the Swedish Medical Research Council No. 5446, Medical Research Council UK, Foundation for Geriatric Research, Uppsala Geriatric Fund, Ernfors Fund for Diabetes Research, "Förenade Liv" Mutual Group Life Insurance Company, The Swedish Diabetes Association, Thureus Foundation and Uppsala University. We thank the technical staff of the Department of Clinical Biochemistry, Addenbrooke's Hospital for the measurements of specific insulin, intact and 32-33-split proinsulin, and Uppsala Clinical Research Centre for excellent statistical support.

\section{References}

1. Beckman JA, Creager MA, Libby P (2002) Diabetes and atherosclerosis: epidemiology, pathophysiology, and management. JAMA 287:2570-2581

2. Howard G, O'Leary DH, Zaccaro D et al (1996) Insulin sensitivity and atherosclerosis. The insulin resistance atherosclerosis study (IRAS) investigators. Circulation 93:1809-1817

3. Laakso M (1993) How good a marker is insulin level for insulin resistance? Am J Epidemiol 137:959-965

4. Despres JP, Lamarche B, Mauriege P et al (1996) Hyperinsulinemia as an independent risk factor for ischemic heart disease. N Engl J Med 334:952-957

5. Reaven GM, Laws A (1994) Insulin resistance, compensatory hyperinsulinaemia, and coronary heart disease. Diabetologia 37:948-952

6. Wingard DL, Barrett-Connor EL, Ferrara A (1995) Is insulin really a heart disease risk factor? Diabetes Care 18:1299-1304
7. Ruige JB, Assendelft WJ, Dekker JM, Kostense PJ, Heine RJ, Bouter LM (1998) Insulin and risk of cardiovascular disease: a meta-analysis. Circulation 97:996-1001

8. Pyorala M, Miettinen H, Laakso M, Pyorala K (1998) Hyperinsulinemia predicts coronary heart disease risk in healthy middle-aged men: the 22-year follow-up results of the Helsinki policemen study. Circulation 98:398-404

9. Kuusisto J, Mykkanen L, Pyorala K, Laakso M (1994) NIDDM and its metabolic control predict coronary heart disease in elderly subjects. Diabetes 43:960-967

10. Yudkin JS, May M, Elwood P, Yarnell JW, Greenwood R, Davey Smith G (2002) Concentrations of proinsulin-like molecules predict coronary heart disease risk independently of insulin: prospective data from the Caerphilly study. Diabetologia 45:327-336

11. Zethelius B, Byberg L, Hales CN, Lithell H, Berne C (2002) Proinsulin is an independent predictor of coronary heart disease: report from a 27-year follow-up study. Circulation 105:21532158

12. Lindahl B, Dinesen B, Eliasson M et al (1999) High proinsulin concentration precedes acute myocardial infarction in a nondiabetic population. Metabolism 48:1197-1202

13. Lindahl B, Dinesen B, Eliasson M, Roder M, Hallmans G, Stegmayr B (2000) High proinsulin levels precede first-ever stroke in a nondiabetic population. Stroke 31:2936-2941

14. DeFronzo RA, Tobin JD, Andres R (1979) Glucose clamp technique: a method for quantifying insulin secretion and resistance. Am J Physiol 237:E214-E223

15. Sobey WJ, Beer SF, Carrington CA, Clark PMS, Hales CN (1989) Sensitive and specific two-site immunoradiometric assays for human insulin, proinsulin, 65-66 split and 32-33 split proinsulins. Biochem J 260:535-541

16. Hedstrand H (1975) A study of middle-aged men with particular reference to risk factors for cardiovascular disease. Uppsala J Med Sci 80(Suppl 19):1-61

17. Byberg L, McKeigue PM, Zethelius B, Lithell HO (2000) Birth weight and the insulin resistance syndrome: association of low birth weight with truncal obesity and raised plasminogen activator inhibitor-1 but not with abdominal obesity or plasma lipid disturbances. Diabetologia 43:54-60

18. Merlo J, Lindblad U, Pessah-Rasmussen H, et al (2000) Comparison of different procedures to identify probable cases of myocardial infarction and stroke in two Swedish prospective cohort studies using local and national routine registers. Eur J Epidemiol 16:235-243

19. Tunstall-Pedoe H, Kuulasmaa K, Amouyel P, Arveiler D, Rajakangas AM, Pajak A (1994) Myocardial infarction and coronary deaths in the World Health Organization MONICA project. Registration procedures, event rates, and case-fatality rates in 38 populations from 21 countries in four continents. Circulation 90:583-612

20. Zethelius B, Hales CN, Lithell HO, Berne C (2004) Insulin resistance, impaired early insulin response, and insulin propeptides as predictors of the development of type 2 diabetes: a population-based, 7-year follow-up study in 70-year-old men. Diabetes Care 27:1433-1438

21. WHO (1999) Definition, diagnosis and classification of diabetes mellitus and its Complications: report of a WHO consultation. World Health Organization: Geneva. WHO/NCD/NCS/99.2

22. Stout RW (1996) Hyperinsulinemia and atherosclerosis. Diabetes 45(Suppl 3):S45-S46

23. Adler AI, Neil HA, Manley SE, Holman RR, Turner RC (1999) Hyperglycemia and hyperinsulinemia at diagnosis of diabetes and their association with subsequent cardiovascular disease in the United Kingdom prospective diabetes study (UKPDS 47). Am Heart J 138:353-359

24. Hanley AJ, Williams K, Stern MP, Haffner SM (2002) Homeostasis model assessment of insulin resistance in relation to the incidence of cardiovascular disease: the San Antonio heart study. Diabetes Care 25:1177-1184 
25. Resnick HE, Jones K, Ruotolo G et al (2003) Insulin resistance, the metabolic syndrome, and risk of incident cardiovascular disease in nondiabetic American Indians: the strong heart study. Diabetes Care 26:861-867

26. Coutinho M, Gerstein HC, Wang Y, Yusuf S (1999) The relationship between glucose and incident cardiovascular events. A metaregression analysis of published data from 20 studies of 95,783 individuals followed for 12.4 years. Diabetes Care 22:233-240

27. Boden G (1997) Role of fatty acids in the pathogenesis of insulin resistance and NIDDM. Diabetes 46:3-10

28. Steinberg HO, Chaker H, Leaming R, Johnson A, Brechtel G, Baron AD (1996) Obesity/insulin resistance is associated with endothelial dysfunction. Implications for the syndrome of insulin resistance. J Clin Invest 97:2601-2610

29. Salonen JT, Lakka TA, Lakka HM, Valkonen VP, Everson SA, Kaplan GA (1998) Hyperinsulinemia is associated with the incidence of hypertension and dyslipidemia in middle-aged men. Diabetes 47:270-275

30. Yudkin JS (2002) Increased proinsulin concentrations and excess risk of coronary heart disease in patients with diabetes and prediabetes. Circulation 106:e202

31. Bavenholm P, Proudler A, Tornvall P et al (1995) Insulin, intact and split proinsulin, and coronary artery disease in young men. Circulation 92:1422-1429

32. Haffner SM, D'Agostino R, Mykkanen L et al (1998) Proinsulin and insulin concentrations in relation to carotid wall thickness: Insulin Resistance Atherosclerosis Study. Stroke 29:1498-1503

33. Galloway JA, Hooper SA, Spradlin CT et al (1992) Biosynthetic human proinsulin. Review of chemistry, in vitro and in vivo receptor binding, animal and human pharmacology studies, and clinical trial experience. Diabetes Care 15:666-692
34. Nordt TK, Schneider DJ, Sobel BE (1994) Augmentation of the synthesis of plasminogen activator inhibitor type-1 by precursors of insulin. A potential risk factor for vascular disease. Circulation 89:321-330

35. Festa A, D. Agostino R J, Mykkanen L et al (1999) Relative contribution of insulin and its precursors to fibrinogen and PAI-1 in a large population with different states of glucose tolerance. The insulin resistance atherosclerosis study (IRAS). Arterioscler Thromb Vasc Biol 19:562-568

36. Kohler HP, Grant PJ (2000) Plasminogen-activator inhibitor type 1 and coronary artery disease. N Engl J Med 342:1792-1801

37. Jehle PM, Lutz MP, Fussgaenger RD (1996) High affinity binding sites for proinsulin in human IM-9 lymphoblasts. Diabetologia 39:421-432

38. Khaw KT, Wareham N, Luben R et al (2001) Glycated haemoglobin, diabetes, and mortality in men in Norfolk cohort of European prospective investigation of cancer and nutrition (EPIC-Norfolk). BMJ 322:15-28

39. Temple RC, Carrington CA, Luzio SD et al (1989) Insulin deficiency in non-insulin-dependent diabetes. Lancet 1:293295

40. Oh JY, Barrett-Connor E, Wedick NM (2002) Sex differences in the association between proinsulin and intact insulin with coronary heart disease in nondiabetic older adults: the Rancho Bernardo study. Circulation 105:1311-1316 